\section{Calcitonin response to naturally occurring ionised \\ hypercalcaemia in cats with chronic kidney disease}

\section{D.H.N. van den Broek ${ }^{1}$, R.F. Geddes', T.L. Williams ${ }^{2}$, Y.M. Chang', R.E. Jepson', J. Elliott ${ }^{1}$}

1 Royal Veterinary College, University of London, London, UK 2 Department of Veterinary Medicine, University of Cambridge, Cambridge, UK

Calcitonin, released by C-cells of the thyroid gland in response to increased serum ionised calcium concentration (iCa), protects against hypercalcaemia by inhibition of osteoclasts, but does not have an important physiologic role in all species. In cats, experimentally-induced hypercalcaemia elicits a calcitonin response in some but not all animals, possibly causing non-responders to be more susceptible to hypercalcaemia. lonised hypercalcaemia is increasingly recognised in cats, some of which have chronic kidney disease (CKD). The aim of this study was to explore the calcitonin response to ionised hypercalcaemia in cats with CKD.

Twenty-eight cats diagnosed with CKD (including 13 cats developing hypercalcaemia within 8 months) were selected for this retrospective observational cohort study. iCa $>1.40 \mathrm{mmol} / \mathrm{L}$ was classified as hypercalcaemia. Calcitonin was measured by a immunoradiometric assay (developed for human samples) on stored heparinised plasma taken at time of CKD diagnosis, 4-9 and 20-33 weeks later for all cases, and at return of normocalcaemia for responders. Groups were compared using independent samples $t$-test or Mann-Whitney $U$ test. Correlations were assessed using Spearman's rank correlation.

Three out of 13 hypercalcaemic cats responded to hypercalcaemia with a calcitonin increase. All other cases had calcitonin levels at all visits that were below the limit of detection of the assay $(1.2 \mathrm{pg} / \mathrm{mL})$. In responders, a trend was observed for plasma calcitonin concentrations to change in parallel with whole blood $\mathrm{iCa}$, but no significant correlation was found. Baseline (range $<1.2-33.6 \mathrm{pg} / \mathrm{mL}$ ) and maximal (range 4.0-87.2 pg/mL) concentrations of calcitonin varied among responders. Maximal iCa during follow-up showed a tendency to be lower in responders than in non-responders (median [range]: 1.46 [1.42-1.46] and 1.52 [1.43-1.73] mmol/L, respectively), although this study lacks statistical power to detect a significant difference $(P=0.141)$.

In conclusion, a subset of cats responded to hypercalcaemia with an increase in calcitonin, where calcitonin concentration seems to change in parallel with ionised calcium concentration. Calcitonin could play a role in the regulation of blood ionised calcium in cats with CKD, but the causeand-effect relationship is unclear. A calcitonin response could possibly limit the severity of hypercalcaemia, but a larger population of cats needs to be studied to test this presumption. Future studies looking at calcitonin and the hypercalcaemic response in cats with CKD are warranted.

\section{Calcitonin response in naturally occurring hypercalcaemia in cats}

\section{Evangelia Maniaki', Carmen Pineda ${ }^{2}$, Zaila Dunbar ${ }^{3}$, Natalie Finch ${ }^{1}$}

University of Bristol, Bristol, UK

University of Cordoba, Cordoba, Spain

Queens Park Petcare, London, UK

Hypercalcaemia stimulates calcitonin synthesis in the C-cells of the thyroid gland in order to restore normocalcaemia by primarily inhibiting osteoclastic bone resorption. A previous study has shown that in some cats with acutely induced hypercalcaemia, calcitonin production is not increased. It has also been suggested in other species that the ability of calcitonin to reduce calcium concentration is related to the degree of bone turnover. This study aims to evaluate calcitonin response in naturally occurring hypercalcaemia in cats.

Hypercalcaemic cats (ionised calcium [ICa] persistently $>1.40 \mathrm{mmol} / \mathrm{l}$ ) presenting to a small animal referral hospital, between 2013 and 2015 were identified retrospectively. Serum was analysed for routine biochemical parameters. Additional diagnostics were performed dependent on case presentation. Serum calcitonin concentration was analysed using a previously validated immunoradiometric assay. Alkaline phosphatase (ALKP) activity was used as a marker of bone turnover. The correlation between ICa and calcitonin and also ALKP activity and both $\mathrm{ICa}$ and calcitonin were examined using Spearman's correlation coefficient (rs). Significance was set at $P<0.05$.

A total of 18 cats were included in the study (13/18 DSH/ DLH, 5/18 pedigree, 11/18 female, 7/18 male, median [range] age 5 [0.5-18] years). Idiopathic hypercalcaemia was diagnosed in 10/18 (56\%) cases, neoplasia in $2 / 18$ (11\%), dietary associated hypervitaminosis D in 2/18 (11\%), granulomatous disease in 1/18 (5\%) and the diagnosis was inconclusive in $3 / 18$ cats (17\%). Median (range) ICa, total calcium and calcitonin was $1.62(1.40-1.62) \mathrm{mmol} / \mathrm{l}, 3.34(2.60-4.0) \mathrm{mmol} / \mathrm{l}$ and $<0.9(<0.9-22.93) \mathrm{pg} / \mathrm{ml}$ respectively. Thirteen cats (72\%) did not have an increased calcitonin concentration above the previously reported reference interval for normocalcaemic cats $(<0.9 \mathrm{pg} / \mathrm{ml})$. All five cats with increased calcitonin concentration had a diagnosis of idiopathic hypercalcaemia. There was no significant correlation between ICa and calcitonin ( $\mathrm{rs}=-0.007, P=0.979$ ), ICa and ALKP activity ( $r S=0.169, P=0.531)$ or calcitonin and ALKP activity ( $r s=-0.019, P=0.470)$.

There was no relationship between ICa and calcitonin concentration in cats with naturally occurring hypercalcaemia. Moreover, the calcitonin concentration was not increased in the majority of cats. This finding questions the concept that calcitonin concentration increases in response to hypercalcaemia in cats. The lack of correlation between ALKP activity and both calcitonin and ICa concentration may suggest either that there is no association with bone turnover or that ALKP activity is not specific for evaluating bone turnover. 